\title{
Anestesia epidural cranial com lidocaína e morfina para campanhas de castração em cães
}

\author{
Cranial epidural anesthesia with lidocaine and morphine for sterilization \\ campaign in dogs
}

\section{Renato Batista Tamanho' ${ }^{\mathrm{I}}$ Nilson Oleskovicz ${ }^{\mathrm{I} *}$ Aury Nunes de Moraes ${ }^{\mathrm{I}}$ Fabíola Niederauer Flôres $^{\mathrm{I}}$

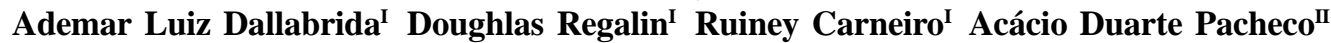 Ademir Cassiano da Rosa ${ }^{\mathrm{I}}$}

\section{RESUMO}

A castração de machos e fêmeas tem sido preconizada como a principal técnica para redução do grande número de cães errantes. No entanto, vários são os entraves com relação à escolha do melhor protocolo anestésico, em relação à eficácia, segurança e redução de custos. Objetivouse, com este trabalho, avaliar os efeitos cardiorrespiratórios, hemogasométricos e analgésicos da utilização de lidocaína em um volume maior, associada à morfina, pela via epidural em cadelas submetidas à ovariosalpingohisterectomia (OSH), com ou sem suplementação de oxigênio. Utilizaram-se 12 cadelas, com peso médio de $11,5 \pm 3,7 \mathrm{~kg}$ e idade de um a quatro anos. Os animais receberam como medicação préanestésica (MPA) acepromazina $\left(0,1 \mathrm{mg} \mathrm{kg}^{-1}\right)$ e meperidina (5mg $\left.\mathrm{kg}^{-1}\right)$, pela via intramuscular. Após 15 minutos, administrou-se tiopental (10mg $\left.\mathrm{kg}^{-1}\right)$, por via intravenosa, seguido de intubação endotraqueal. Nesse momento, os animais foram alocados em dois grupos: o grupo GCO (com oxigênio, $n=06$ ) recebeu suplementação de oxigênio $100 \%$ e o grupo GSO (sem oxigênio, $n=06$ ) permaneceu intubado sem suplementação de oxigênio. Após a intubação, foi administrada, pela via epidural, em ambos os grupos, morfina $\left(0,1 \mathrm{mg} \mathrm{kg}^{-1}\right)$ em volume final ajustado para $1 \mathrm{~mL} 3,0 \mathrm{~kg}^{-1}$ de peso com lidocaína $2 \%$ sem vasoconstritor. Imediatamente após a anestesia epidural, os animais foram posicionados em decúbito dorsal com a cabeça no mesmo plano do corpo, e iniciou-se o procedimento cirúrgico, o qual foi padronizado em 30 minutos. Em ambos os grupos, foi possível realizar a cirurgia sem a necessidade de complementação analgésica e sem resposta de dor. A pressão arterial média (PAM) foi menor no GSO em todos os momentos em comparação ao basal. No GCO, a PAM foi menor após MPA e após epidural. Houve redução da fe $\mathrm{M} 1$ até $\mathrm{M} 6$ no GSO. A $\mathrm{SaO}_{2}$ e $\mathrm{PaO}_{2}$ foram maiores no GCO em comparação ao GSO. O pH foi menor no
GCO 15 minutos após MPA até 40 minutos após epidural, em comparação ao GSO. Conclui-se que a anestesia epidural lombossacra com morfina e lidocaína na dose e no volume propostos é efetiva para realização de OSH em cadelas, com mínimas alterações cardiovasculares e hemogasométricas, as quais são bem toleradas em animais hígidos. Essa prática é exequível em campanhas de castração em que não há possibilidade de oxigenação dos animais.

Palavras-chave: cães, morfina, epidural, campanhas de castração.

\section{ABSTRACT}

Male and female sterilization have been established as the main technique to reduce the huge number of mongrel dogs. However, there are several barriers regarding to the choice of the best anesthetic protocol, in terms of efficacy, security and cost reduction. The aim of this study was to evaluate the analgesic, cardiorespiratory and hemogasometric effects of epidural anesthesia with a large volume of lidocaine in combination with morphine in female dogs submitted to ovariosalpingohisterectomy (OSH), with or without oxygen supplementation. Twelve adult female dogs were used, with average weight of $11.5 \pm 3.7 \mathrm{~kg}$ and age of 1 to 4 years. The animals received acepromazine $\left(0.1 \mathrm{mg} \mathrm{kg}^{-1}\right)$ and meperidine (5mg $\left.\mathrm{kg}^{-1}\right)$ as premedication, administered by the intramuscular route. Fifteen minutes later, thiopental (10 $\left.\mathrm{mg} \mathrm{kg}^{-1}\right)$ was administered by intravenous route, followed by endotracheal intubation. The animals were allocated into two groups: GCO (group with oxygen, $n=06$ ), where the animals received $100 \%$ oxygen supplementation and GSO (group without oxygen supplementation, $n=6)$, mantained with endotracheal intubation, but without oxygen supplementation. After intubation, epidural with morphine $\left(0,1 \mathrm{mg} \mathrm{kg} \mathrm{g}^{-1}\right)$, adjusted to

IDepartamento de Medicina Veterinária, Universidade do Estado de Santa Catarina (UDESC). Av. Luiz de Camões 2090, Bairro Conta Dinheiro, 88520-000, Lages, SC, Brasil. E-mail: a2no@cav.udesc.br. *Autor para correspondência.

IDepartamento de Clínica, Cirurgia e Reprodução Animal, Faculdade de Odontologia de Araçatuba (FOA), Universidade Estadual Paulista (UNESP), Araçatuba, SP, Brasil. 
$1 \mathrm{~mL} 3 \mathrm{~kg}^{-1}$ in lidocaine $2 \%$ without epinepherine was administered. Immediately after epidural administration, the animals were positioned in dorsal recumbency, with the head in the same level of the body. In both groups, surgery was performed without the need of additional analgesia and without signs of pain. Mean arterial pressure (MAP) was lower in GSO in all moments compared to baseline values. In GCO, MAP was lower after premedication and epidural anesthesia. Respiratory rate was lower from $\mathrm{M} 1$ to $\mathrm{M} 6$ in GSO. The $\mathrm{SaO}_{2}$ and $\mathrm{PaO}_{2}$ were higher in GCO when compared to GSO. The $\mathrm{pH}$ was lower in GCO after 15 minutes after premedication, until 40 minutes after the epidural administration, compared to GSO. It was concluded that the epidural administration using morphine and lidocaine in the dosage and volume proposed are effective to $\mathrm{OSH}$ procedure in dogs. The lack of oxygen supplementation promoted minimal cardiovascular and hemogasometric alterations, which is feasible in sterilizations campaigns where there is no possibility of oxygen supplementation of the animals.

Key words: dogs, morphine, epidural, sterilizations campaigns.

\section{INTRODUÇÃO}

Em virtude do aumento do número de animais errantes encontrados nas grandes cidades, da proliferação de doenças e zoonoses vinculadas a esse processo, além do aumento considerável de animais de estimação presentes nas regiões mais carentes dos municípios, busca-se encontrar soluções rotineiras, eficientes e economicamente viáveis para solucionar ou amenizar o problema. Devido a isso, prefeituras, em parceria com universidades e organizações não governamentais (ONGS), vêm buscando cada vez mais programas de castração em massa com o intuito de reduzir o número de animais de rua (DINIZ, 2009).

Para tanto, se torna necessária a utilização de protocolos anestésicos que sejam de baixo custo, práticos e promovam analgesia adequada, tanto trans, como pós-operatória. Nesse contexto, destaca-se a anestesia epidural como uma técnica anestésica consagrada, que tem como principais vantagens: segurança, eficiência e baixo custo (CRUZ et al., 1997). Além disso, o fármaco injetado por essa via sofre menor absorção e, portanto, acarreta efeitos sistêmicos menos pronunciados (PASCOE, 1992).

A anestesia epidural com anestésicos locais possui baixa seletividade, produzindo, tanto bloqueio sensitivo, como bloqueio motor, tornando essa técnica pouco atrativa para o controle da dor por períodos prolongados (TORSKE et al., 1998). Além disso, o uso isolado de anestésicos locais, normalmente lidocaína, não é suficiente para produzir anestesia que viabilize a ovariosalpingohisterectomia (OSH) em cães (ISHIY et al., 2002), já que os ovários são inervados pelo terceiro e pelo quarto nervo lombar (BAILEY et al., 1988), e a utilização da lidocaína via epidural na dose usual de $5 \mathrm{mg} \mathrm{kg}^{-1}$ ou $0,26 \mathrm{~mL} \mathrm{~kg}^{-1}$ determina bloqueio anestésico máximo entre a quarta ou a quinta vértebra lombar (ISHIY et al., 2002; GASPARINI et al., 2007).

Estudos sobre a fisiologia da dor, particularmente da dor clínica, têm fomentado o uso de agentes analgésicos opioides como importante alternativa no controle da nocicepção em animais e, principalmente pela via epidural, para o controle segmentar da dor pós-operatória (POPILSKIS et al., 2000), produzindo analgesia pós-cirúrgica de melhor qualidade e maior duração (de 16 a 24 horas) (BONATH \& SALEH 1985) do que aquela obtida após administração desses agentes por via parenteral. Nesse sentido, a administração epidural de opioides promove alívio da dor visceral e somática pelo bloqueio seletivo de impulsos nociceptivos, sem interferir nas funções sensorial e motora, bloqueando o estímulo doloroso no corno dorsal da medula espinhal, por inibição das vias somatossensoriais aferentes e supraespinhais e ativação das vias inibitórias descendentes (THURMON et al., 2007).

Dentre os opioides utilizados pela via epidural em cães, a morfina destaca-se pela ação analgésica duradoura e pelos mínimos efeitos colaterais (PASCOE, 1992). A administração lombossacra de morfina é recomendada para intervenções na região caudal do corpo. Entretanto, existem evidências de que a analgesia possa se estender rostralmente em direção ao tórax e aos membros torácicos (VALVERDE et al., 1989; PASCOE \& DYSON, 1993;).

Segundo THURMON et al. (2007), a aplicação epidural de $1 \mathrm{mg}$ total de morfina, diluída em 3 a $4 \mathrm{ml}$ de solução salina, avança até o quarto ou quinto espaço lombar, em cães com $10-15 \mathrm{~kg}$, produzindo analgesia de toda a região caudal ao arco costal, por aproximadamente 22 horas, sem afetar a frequência cardíaca, a pressão arterial, o débito cardíaco, a resistência vascular periférica, a $\mathrm{PaCO}_{2}, \mathrm{a} \mathrm{PaO}_{2}$ e o pH arterial.

A injeção simultânea de anestésicos locais e opioides oferece como vantagens o rápido início de ação do anestésico local, produzindo bloqueio sensitivo e motor imediato, aliviando a dor e possibilitando, posteriormente, a ação analgésica prolongada do opioide. Tais associações, por atuarem em diferentes locais e receptores, são alternativas que minimizam os efeitos colaterais, às vezes observados com o uso isolado da morfina, e contribuem para melhorar a recuperação do animal no período póscirúrgico (VALADÃO et al., 2002). 
Vários protocolos de anestesia epidural já foram testados e aprovados para uso em cães, inclusive a associação de lidocaína e morfina, sendo uma técnica rotineira para controle da dor, tanto trans-operatória quanto pós-operatória. A maioria desses estudos padronizam o volume final de fármaco injetado pela via epidural em $0,26 \mathrm{~mL} \mathrm{~kg}^{-1}$ ou $1 \mathrm{~mL}$ para cada $4,0 \mathrm{~kg}$ de peso (VALVERDE et al., 1989), $1 \mathrm{~mL}$ para cada $4,5 \mathrm{~kg}$ de peso (BRANSON et al., 1993) ou ainda $1,7 \mathrm{~mL}$ para cada $4 \mathrm{~kg}$ de peso (CASSU et al., 2008). No entanto, não existem relatos da possibilidade de realização de $\mathrm{OSH}$ em cadelas, sem suplementação analgésica adicional.

Outro problema observado nas campanhas de castração em massa é a dificuldade de suplementação de oxigênio, visto que, muitas vezes, trabalha-se com um grande número de cães, com estrutura limitada e com restrições orçamentárias. Dessa forma, torna-se necessário a adequação dos protocolos anestésicos comumente utilizados na rotina em centros de referência.

Dessa forma, objetivou-se avaliar os efeitos cardiovasculares, respiratórios, hemogasométricos e analgésicos da administração de um volume elevado de lidocaína $2 \%$ associada à morfina pela via epidural em cadelas submetidas à ovariosalpingohisterectomia, com ou sem suplementação de oxigênio. Essa técnica vem sendo utilizada rotineiramente pelos autores deste trabalho em campanhas de castração. No entanto, não existem estudos que demonstrem e comprovem a confiabilidade desse procedimento.

\section{MATERIAL E MÉTODOS}

Foram utilizadas 12 cadelas, sem raça definida (SRD), com peso médio de $11,5 \pm 3,7 \mathrm{~kg}$, idade entre um a quatro anos, comprovadamente hígidas e provenientes do Centro de Controle de Zoonoses do Município de Lages, Santa Catarina (SC). Os animais foram desverminados, vacinados e mantidos em gaiolas individuais por 15 dias para padronização dos cuidados e da alimentação. Antes do início do experimento, foram realizados hemograma completo e avaliação de proteínas totais, sendo excluídos os animais comprometidos. No dia do experimento, os animais foram submetidos a jejum alimentar de 12 horas e hídrico de 6 horas. Após esse período, as cadelas foram posicionadas sobre colchão térmico ativo, em decúbito lateral direito, e anestesiadas com isofluorano ${ }^{\mathrm{a}} \mathrm{em}$ vaporizador calibrado mensurado por meio de analisador de gases anestésicos, sendo padronizada a concentração expirada de isofluorano em $3 \mathrm{~V} \%$, administrado por máscara facial por meio de circuito anestésico de Bain, com fluxo diluente de oxigênio de
4L $\min ^{-1}$. Em seguida, foi realizada tricotomia da região ventral do abdômen, região lombossacra, região da veia cefálica e artéria femoral. Após, foi realizado botão anestésico com lidocaína $2 \%$ sem vasoconstritor ${ }^{\mathrm{b}}$ e foi realizada a dissecação da artéria femoral para a introdução de uma sonda urinária, estéril $n^{\circ} 4$ acoplada a um transdutor ${ }^{c}$ de pressão preenchido com solução heparinizada para permitir a mensuração da pressão arterial e a obtenção das amostras de sangue arterial para hemogasometria ${ }^{\mathrm{d}}$. Um cateter $20 \mathrm{G}$ foi introduzido na veia cefálica esquerda para administração de fluidoterapia com solução de Ringer com lactato $(10 \mathrm{~mL}$ $\left.\mathrm{kg}^{-1} \mathrm{~h}^{-1}\right)$. A temperatura da sala foi mantida em $23^{\circ} \mathrm{C}$. O período total de instrumentalização foi de aproximadamente 10 minutos.

Após a preparação dos animais, encerrouse a administração de isofluorano e aguardou-se um período de 30 minutos para recuperação completa destes. Nesse momento, os animais receberam como medicação pré-anestésica (MPA) acepromazina ${ }^{\mathrm{e}}(0,1 \mathrm{mg}$ $\left.\mathrm{kg}^{-1}\right)$ e meperidina ${ }^{\mathrm{f}}\left(5 \mathrm{mg} \mathrm{kg}^{-1}\right)$, pela via intramuscular. Decorridos 15 minutos, administrou-se tiopental sódico ${ }^{g}$ $\left(10 \mathrm{mg} \mathrm{kg}^{-1}\right)$ por via intravenosa, seguido de intubação endotraqueal com endotubo adequado ao tamanho de cada um deles. Nesse momento, os animais foram alocados em dois grupos: $\mathrm{GCO}$ (com oxigênio, $\mathrm{n}=06$ ), os quais receberam suplementação de oxigênio $100 \%$ no volume de $150 \mathrm{ml} \mathrm{kg}^{-1}$ por meio de circuito anestésico de Bain e GSO (sem oxigênio, $n=06$ ), os quais permaneceram intubados sem suplementação de oxigênio.

Após a intubação, os animais foram posicionados em decúbito esternal, com os membros pélvicos estendidos cranialmente para realização da anestesia epidural lombossacra com agulha hipodérmica 40x8. Os animais de ambos os grupos receberam morfina ${ }^{\mathrm{h}}\left(0,1 \mathrm{mg} \mathrm{kg}^{-1}\right)$ com volume final ajustado de $1 \mathrm{~mL}$ para cada $3,0 \mathrm{~kg}$ de peso com lidocaína $2 \%$ sem vasoconstritor, administrado no período de 60 segundos. O correto posicionamento da aplicação epidural foi confirmado pela ausência de resistência à aplicação de ar com auxílio de uma seringa de vidro (THURMON et al., 2007).

Após a realização da anestesia epidural, os animais foram imediatamente colocados em decúbito dorsal com a cabeça posicionada no mesmo nível do corpo, sem elevação, para uma maior progressão do bloqueio anestésico. Aguardou-se um período de cinco minutos para o início do procedimento cirúrgico, o qual foi padronizado em um período de 30 minutos. Nesse momento, foi administrado antibiótico profilático com ampicilina sódica na dose de $20 \mathrm{mg} \mathrm{kg}^{-1}$ pela via intravenosa. 
Foram coletados os seguintes parâmetros: frequência cardíaca (FC), frequência respiratória (f), pressão arterial sistólica (PAS), diastólica (PAD) e média (PAM), temperatura retal por meio de termômetro digital $^{c}$ (TR), temperatura da sala (TS), $\mathrm{PaCO}_{2}, \mathrm{PaO}_{2}$, $\mathrm{SaO}_{2}, \mathrm{pH}, \mathrm{Na}^{+}, \mathrm{K}^{+}, \mathrm{Ca}^{+}, \mathrm{HCO}_{3}^{-}$e déficit de base, nos momentos M-15 (basal), M0 (15 minutos após MPA), M1 (após intubação), M2 (imediatamente após epidural), M3, M4, M5 e M6 (10, 20, 30 e 40 minutos após epidural). O procedimento cirúrgico foi iniciado imediatamente após M3, perdurando até M6. Além disso, foram avaliados parâmetros subjetivos durante a realização do procedimento, como: grau de relaxamento muscular (0: sem relaxamento; 1: leve; 2 : moderado; 3 ótimo), silêncio abdominal (presente ou ausente) e qualidade de recuperação (suave ou com excitação) aferidos sempre pelo mesmo avaliador.

Um aumento da FC e PAS superior a $20 \%$ do valor referente ao momento anterior seria considerado sinal positivo para dor e, nesse caso, administrar-se-ia uma dose resgate de analgésico (fentanili, $4,4 \mu \mathrm{g} \mathrm{kg}^{-1}$, IV). No período pós-operatório, administrou-se enrofloxacina $10 \%$ na dose de $5,0 \mathrm{mg} \mathrm{kg}^{-1}$, por via subcutânea (SC), duas vezes ao dia (BID), durante quatro dias, meloxicam $0,2 \%$ na dose $0,2 \mathrm{mg} \mathrm{kg}^{-1}$, no primeiro dia, e $0,1 \mathrm{mg} \mathrm{kg}^{-1}$ nos três dias subsequentes, $\mathrm{SC}$ e SID. Os dados paramétricos foram submetidos ao teste t entre os grupos e à Análise de Variância, com Repetições Múltiplas entre os tempos dentro de cada grupo $(\mathrm{P} \leq 0,05)$.

\section{RESULTADOS E DISCUSSÃO}

Com relação aos parâmetros subjetivos, todos os animais receberam escore de relaxamento muscular igual a três (ótimo), com silêncio abdominal presente e qualidade de recuperação suave. Não foi necessária a aplicação de fentanil durante o transoperatório em nenhum dos animais, pois não houve aumento da FC e PAS superior a $20 \%$ do valor referente ao momento anterior. Segundo BONATH \& SALEH (1985), a anestesia epidural realizada com o uso de morfina na dose de $0,1 \mathrm{mg} \mathrm{kg}^{-1}$ apresenta um período de latência de 20 a 60 minutos e uma duração que varia entre 16 e 24 horas. Nesse sentido, em virtude do tempo decorrido entre a anestesia epidural e o início da cirurgia, pode-se inferir algum efeito analgésico da morfina durante o terço final da cirurgia. Por outro lado, o objetivo primário da administração desta foi de fornecer ao animal analgesia pós-operatória, visto que o procedimento cirúrgico teve início cinco minutos após a realização da anestesia epidural e duração máxima de 30 minutos. Nesse período, o efeito analgésico da morfina ainda não havia sido alcançado, mas o efeito da lidocaína epidural encontrava-se no pico máximo.

A comprovação da eficiência dessas dosagens e volumes pôde ser constatada pela ausência de dor durante o procedimento cirúrgico, mesmo durante a tração dos ligamentos suspensores do ovário. Tal fato deve-se, em primeiro lugar, ao volume de lidocaína administrado, ou seja, $1 \mathrm{~mL}$ para cada $3 \mathrm{~kg}$ de peso, e também devido à associação de morfina, pois, segundo WETMORE \& GLOWASKI (2000), além do efeito analgésico prolongado, a associação de opioides a anestésicos locais pela via epidural visa a aumentar a duração do bloqueio sensitivo e estendêlo a segmentos espinhais mais craniais. Da mesma forma, NOLTE et al. (1983) demonstraram que bloqueios epidurais craniais que chegam à região espinhal T5 não geram depressão e mudanças no sistema cardiovascular, no f, no $\mathrm{pH}$, na $\mathrm{PaCO}_{2}$ e na $\mathrm{PaO}_{2}$ em cães sedados com acepromazina. Por outro lado, ISHIY et al. (2002) demonstraram que o uso isolado de anestésico local, em doses usuais de $5 \mathrm{mg} \mathrm{kg}^{-1}(0,26 \mathrm{~mL}$ $\left.\mathrm{kg}^{-1}\right)$, normalmente lidocaína, não é suficiente para produzir anestesia que viabilize a $\mathrm{OSH}$ em cães, já que os ovários são inervados pelo terceiro e pelo quarto par de nervo lombar (BAILEY et al., 1988), e o anestésico local, quando administrado em volumes de até $0,26 \mathrm{~mL}$ $\mathrm{kg}^{-1}$, chega somente até o quarto e o quinto espaço lombar.

Não foram observadas diferenças significativas entre os tempos dentro de cada grupo, bem como entre os grupos, para os valores de TS, $\mathrm{Na}^{+}$, $\mathrm{K}^{+}, \mathrm{Ca}^{+}, \mathrm{HCO}_{3}^{-}$e DB. Também não foram observadas diferenças significativas entre os grupos para os valores de FC, PAS, PAD, PAM e TR.

Houve redução significativa da $\mathrm{FC}$ entre os momentos M4 (20 minutos após a anestesia epidural) até M6 (40 minutos após anestesia epidural), em relação ao M-15 (basal), no GSO (Tabela 1). Embora os valores basais de FC não tenham diferido significativamente entre os grupos, valores mais elevados foram observados em GSO. É possível que esses resultados possam explicar o fato de que FC diminuiu significativamente ao longo do tempo em GSO, mas não em GCO. No entanto, observa-se que os valores médios observados estão dentro dos valores normais para cães durante anestesia geral (THURMON et al., 2007). No grupo GSO, observou-se redução significativa nos valores de PAS em todos os momentos quando comparados ao momento basal (M-15). No grupo GCO, ocorreu redução na PAS, nos momentos M0, M2 e M3, quando comparados ao basal. Houve diminuição nos valores de PAD, nos tempos M0, M2 e M3 do grupo GSO, quando comparados ao momento 
Anestesia epidural cranial com lidocaína e morfina para campanhas de castração em cães.

Tabela 1 - Valores médios e desvios-padrão da pressão arterial média (PAM), da sistólica (PAS) e da diastólica (PAD), frequência cardíaca $(\mathrm{FC})$, frequência respiratória $(f)$, pressão parcial de dióxido de carbono arterial $\left(\mathrm{PaCO}_{2}\right)$, pressão parcial de oxigênio arterial $\left(\mathrm{PaO}_{2}\right)$, saturação de oxigênio na hemoglobina do sangue arterial $\left(\mathrm{SaO}_{2}\right)$, potencial hidrogeniônico $(\mathrm{pH})$ e temperatura retal (TR) após a realização da anestesia epidural cranial com morfina e lidocaína para o grupo sem suplementação de oxigênio (GSO) e com suplementação de oxigênio (GCO) em cadelas submetidas à ovariosalpingohisterectomia.

\begin{tabular}{|c|c|c|c|c|c|c|c|c|c|}
\hline Variável & Grupo & M-15 & M0 & M1 & M2 & M3 & M4 & M5 & M6 \\
\hline \multirow{2}{*}{$\begin{array}{l}\text { PAM } \\
(\mathrm{mmHg})\end{array}$} & GCO & $108 \pm 11$ & $82 \pm 14 \mathrm{~A}$ & $99 \pm 9$ & $86 \pm 6 \mathrm{~A}$ & $88 \pm 11$ & $101 \pm 11$ & $99 \pm 16$ & $106 \pm 18$ \\
\hline & GSO & $117 \pm 9$ & $87 \pm 7 \mathrm{~A}$ & $102 \pm 4 \mathrm{~A}$ & $86 \pm 15 \mathrm{~A}$ & $87 \pm 10 \mathrm{~A}$ & $102 \pm 8 \mathrm{~A}$ & $101 \pm 6 \mathrm{~A}$ & $100 \pm 6 \mathrm{~A}$ \\
\hline \multirow{2}{*}{$\begin{array}{l}\text { PAS } \\
(\mathrm{mmHg})\end{array}$} & GCO & $139 \pm 31$ & $108 \pm 9 \mathrm{~A}$ & $118 \pm 7$ & $104 \pm 7 \mathrm{~A}$ & $105 \pm 17 \mathrm{~A}$ & $120 \pm 8$ & $117 \pm 15$ & $124,1 \pm 17$ \\
\hline & GSO & $135 \pm 8$ & $106 \pm 5 \mathrm{~A}$ & $120 \pm 8 \mathrm{~A}$ & $103 \pm 16 \mathrm{~A}$ & $110 \pm 14 \mathrm{~A}$ & $123 \pm 6 \mathrm{~A}$ & $117 \pm 6 \mathrm{~A}$ & $119 \pm 6 \mathrm{~A}$ \\
\hline \multirow{2}{*}{$\begin{array}{l}\text { PAD } \\
(\mathrm{mmHg})\end{array}$} & GCO & $85 \pm 11$ & $67 \pm 7$ & $84 \pm 11$ & $76 \pm 7$ & $76 \pm 13$ & $92 \pm 14$ & $88 \pm 21$ & $93 \pm 19$ \\
\hline & GSO & $97 \pm 13$ & $72 \pm 6 \mathrm{~A}$ & $90 \pm 5$ & $75 \pm 15 \mathrm{~A}$ & $76 \pm 6 \mathrm{~A}$ & $91 \pm 7$ & $92 \pm 7$ & $87 \pm 4$ \\
\hline \multirow{2}{*}{$\begin{array}{l}\text { FC } \\
\left(\text { bat } \min ^{-1}\right)\end{array}$} & GCO & $110 \pm 6$ & $98 \pm 14$ & $115 \pm 19$ & $96 \pm 17$ & $96 \pm 22$ & $91 \pm 23$ & $97 \pm 14$ & $102 \pm 20$ \\
\hline & GSO & $121 \pm 26$ & $105 \pm 19$ & $113 \pm 17$ & $111 \pm 29$ & $108 \pm 14$ & $91 \pm 19 \mathrm{~A}$ & $95 \pm 11 \mathrm{~A}$ & $94 \pm 14 \mathrm{~A}$ \\
\hline \multirow{2}{*}{$\begin{array}{l}f \\
\left(\operatorname{mov} \min ^{-1}\right)\end{array}$} & GCO & $26 \pm 7$ & $20 \pm 6$ & $18 \pm 7$ & $16 \pm 4$ & $16 \pm 6$ & $18 \pm 7$ & $24 \pm 10$ & $21 \pm 8$ \\
\hline & GSO & $31 \pm 6$ & $30 \pm 6$ & $22 \pm 4 \mathrm{~A}$ & $20 \pm 3 \mathrm{~A}$ & $17 \pm 2 \mathrm{~A}$ & $17 \pm 3 \mathrm{~A}$ & $16 \pm 2 \mathrm{~A}$ & $17 \pm 2 \mathrm{~A}$ \\
\hline \multirow{2}{*}{$\begin{array}{l}\mathrm{PaCO}_{2} \\
(\mathrm{mmHg})\end{array}$} & GCO & $31,6 \pm 5$ & $34,5 \pm 5$ & $40,9 \mathrm{~A} \pm 8$ & $38,2 \pm 7$ & $36,4 \pm 6$ & $38,4 \pm 5$ & $37,2 \pm 5$ & $36,1 \pm 5$ \\
\hline & GSO & $29,3 \pm 3$ & $34,1 \pm 2$ & $34,5 \pm 5$ & $31,3 \pm 6$ & $32,2 \pm 4$ & $34,6 \pm 2$ & $33,6 \pm 4$ & $31,8 \pm 6$ \\
\hline \multirow{2}{*}{$\begin{array}{l}\mathrm{PaO}_{2} \\
(\mathrm{mmHg})\end{array}$} & GCO & $94,4 \pm 9$ & $87,2 \pm 8$ & $381,7 \pm 65 \mathrm{Aa}$ & $450,0 \pm 77 \mathrm{Aa}$ & $470,8 \pm 55 \mathrm{Aa}$ & $496,0 \pm 59$ & $450,8 \pm 63 \mathrm{Aa}$ & $473,6 \pm 73 \mathrm{Aa}$ \\
\hline & GSO & $107,0 \pm 17$ & $85,8 \pm 6 \mathrm{~A}$ & $76,7 \pm 9 \mathrm{Ab}$ & $89,3 \pm 18 \mathrm{Ab}$ & $91,4 \pm 15 \mathrm{Ab}$ & $82,7 \pm 4 \mathrm{Ab}$ & $92,0 \pm 10 \mathrm{Ab}$ & $97,3 \pm 10 \mathrm{Ab}$ \\
\hline \multirow{2}{*}{$\begin{array}{l}\mathrm{SaO}_{2} \\
(\%)\end{array}$} & $\mathrm{GCO}$ & $96,8 \pm 0,9$ & $96,2 \pm 1,0$ & $99,6 \pm 0,3 \mathrm{Aa}$ & $99,8 \pm 0,1 \mathrm{Aa}$ & $99,7 \pm 0,3 \mathrm{Aa}$ & $99,7 \pm 0,3 \mathrm{Aa}$ & $99,8 \pm 0,1 \mathrm{Aa}$ & $99,7 \pm 0,3 \mathrm{Aa}$ \\
\hline & GSO & $97,6 \pm 0,9$ & $95,0 \pm 2,6 \mathrm{~A}$ & $93,3 \pm 4,1 \mathrm{Ab}$ & $95,8 \pm 1,8 \mathrm{Ab}$ & $96,3 \pm 1,5 \mathrm{Ab}$ & $96,1 \pm 1,1 \mathrm{Ab}$ & $96,9 \pm 1,0 \mathrm{~b}$ & $97,2 \pm 0,6 b$ \\
\hline \multirow{2}{*}{$\mathrm{pH}$} & GCO & $7,40 \pm 0,03$ & $7,37 \pm 0,01 \mathrm{a}$ & $7,32 \pm 0,02 \mathrm{Aa}$ & $7,35 \pm 0,03 \mathrm{a}$ & $7,36 \pm 0,03 \mathrm{a}$ & $7,33 \pm 0,07 \mathrm{a}$ & $7,35 \pm 0,04 \mathrm{a}$ & $7,38 \pm 0,02 \mathrm{a}$ \\
\hline & GSO & $7,42 \pm 0,03$ & $7,39 \pm 0,01 \mathrm{~b}$ & $7,39 \pm 0,03 b$ & $7,39 \pm 0,02 \mathrm{Ab}$ & $7,38 \pm 0,02 \mathrm{Aa}$ & $7,36 \pm 0,01 \mathrm{Aa}$ & $7,38 \pm 0,01 \mathrm{Aa}$ & $7,38 \pm 0,02 \mathrm{Aa}$ \\
\hline TR & $\mathrm{GCO}$ & $38,5 \pm 0,3$ & $38,1 \pm 0,4$ & $37,4 \pm 0,4 \mathrm{~A}$ & $37,5 \pm 0,3 \mathrm{~A}$ & $37,3 \pm 0,6 \mathrm{~A}$ & $37,1 \pm 0,8 \mathrm{~A}$ & $36,9 \pm 0,9 \mathrm{~A}$ & $37,0 \pm 0,9 \mathrm{~A}$ \\
\hline$(? \mathrm{C})$ & GSO & $38,5 \pm 0,5$ & $38,4 \pm 0,5$ & $38,0 \pm 0,6$ & $37,8 \pm 0,7 \mathrm{~A}$ & $37,8 \pm 0,6 \mathrm{~A}$ & $37,8 \pm 0,6 \mathrm{~A}$ & $37,6 \pm 0,6 \mathrm{~A}$ & $37,5 \pm 0,6 \mathrm{~A}$ \\
\hline
\end{tabular}

Letras maiúsculas nas linhas significam diferença de $\mathrm{M}-15(\mathrm{P}=0,05)$. Letras minúsculas diferentes nas colunas significam diferença entre grupos $(\mathrm{P}=0,05)$.

basal. A PAM reduziu em todos os momentos no GSO, quando comparado ao basal (Tabela 1). No grupo GCO, ocorreu redução nos valores de PAM, nos momentos M0 e M2, quando comparados ao basal. Observou-se, no presente estudo, que a administração epidural de um volume elevado de lidocaína associada à morfina não resultou em redução adicional na pressão arterial além daquela observada antes da anestesia epidural (M0 e M1). Os efeitos sobre a pressão arterial observados previamente à anestesia epidural parecem ter sido ocasionados pela administração da acepromazina, assim como descrito por FARVER et al. (1986). Por outro lado, CARPENTER et al. (1992) afirmam que a aplicação de doses adicionais ou maiores de anestésicos locais tem sido associada com bloqueio simpático, hipotensão, paralisia e hipotermia.
A TR foi reduzida no GSO do M2 até M6, em comparação com a M-15, fato que também ocorreu no GCO a partir de M1 até M6 (Tabela 1). A discreta redução da temperatura retal no decorrer do tempo pode ser atribuída à ação vasodilatadora e depressora dos mecanismos termorreguladores do hipotálamo e miorrelaxamento induzido pela acepromazina (HALL \& CLARKE, 1991). Além disso, a anestesia epidural também pode ser prejudicial ao controle da termorregulação central e periférica, induzindo a ocorrência de hipotermia (MATSUKAWA et al., 1995). Ademais, durante a cirurgia, outros fatores facilitam a ocorrência da redução da temperatura corpórea, como exposição da cavidade abdominal, perda sanguínea e anestesia geral (CASSU et al., 2008). A temperatura retal foi reduzida pela anestesia geral, em todos os grupos. 
Porém, durante a maior parte do estudo, a TR em ambos os grupos foi mantida em valores iguais ou maiores a $37^{\circ} \mathrm{C}$, não caracterizando hipotermia (WATERMAN, 1975).

Observou-se redução da $\mathrm{f}$ em relação ao basal no GSO de M1 até M6, mas não no GCO. Tal fato provavelmente ocorreu em virtude dos maiores valores obtidos no tempo basal (M-15), no GSO. No entanto, os valores obtidos estão dentro dos valores físiológicos para a espécie (Tabela 1). Além disso, não foram observadas diferenças entre os grupos estudados. VALVERDE et al. (1989) afirmam que os efeitos benéficos e colaterais da injeção epidural de opioides podem estar relacionados ao volume final da solução, embora não observaram diferenças significativas na analgesia segmentar obtida em cães, após injetar morfina $(0,1 \mathrm{mg}$ $\left.\mathrm{kg}^{-1}\right)$, tanto diluída num volume de $0,13 \mathrm{~mL} \mathrm{~kg}^{-1}$ ou em $0,26 \mathrm{~mL} \mathrm{~kg}^{-1}$. Cabe salientar que ambos os grupos receberam tiopental sódico como agente indutor único para promover maior conforto ao anestesista e ao animal no momento da realização da anestesia epidural e este promove diminuição da f após sua administração, em virtude de sua ação depressora sobre o centro respiratório bulbar, acarretando hipoventilação e consequentemente elevação da $\mathrm{PaCO}_{2}$ (THURMON et al., 2007; MUIR III et al., 2001).

$\mathrm{A} \mathrm{SaO}_{2}$ foi significativamente menor no $\mathrm{GSO}$, nos momentos de M0 até M4, quando comparados ao M-15. Em contrapartida, no GCO, ocorreu um aumento expressivo a partir de M1 até M6, em comparação com a M-15 (Tabela 1). Na análise entre grupos, foram constatados valores significativamente maiores da $\mathrm{SaO}_{2}$ no grupo GCO, quando comparado a GSO de M1 até M6. Essas alterações são facilmente explicadas, pois os animais do GCO foram suplementados com oxigênio $100 \%$, ou seja, tiveram uma fração inspirada de oxigênio $\left(\mathrm{FiO}_{2}\right)$ de 1; dessa maneira apresentaram valores de $\mathrm{PaO}_{2}$ maiores desde a intubação até o final do período de avaliação e, consequentemente, valores maiores de $\mathrm{SaO}_{2}$.

Ocorreu uma redução significativa nos valores da $\mathrm{PaO}_{2}$, no grupo GSO, em todos os momentos em relação ao basal. Em contrapartida, no GCO, ocorreu um aumento expressivo nos valores de $\mathrm{PaO}_{2}$ a partir de M1 até M6, em comparação ao basal. Na análise entre grupos, foram observados valores significativamente maiores na $\mathrm{PaO}_{2}$ do grupo GCO em relação ao GSO, a partir de M1 até M6. Esses resultados ocorreram em decorrência da suplementação de oxigênio no GCO (Tabela 1). Ressalta-se que todos os valores de $\mathrm{PaO}_{2}$ obtidos para o grupo GSO foram superiores a $68 \mathrm{mmHg}$, o qual, de acordo com a curva de dissociação da oxihemoglobina para o cão, resulta em valores de $\mathrm{SaO}_{2}$ iguais ou maiores a 90\%. Esses valores de $\mathrm{PaO}_{2}$ e $\mathrm{SaO}_{2}$ são menores do que aqueles considerados normais para o cão e são esperados para um paciente sob anestesia geral, sem suplementação de oxigênio, baseado no fato de serem submetidos a uma $\mathrm{FiO}_{2}$ de 0,21.

Não foi observada alteração significativa entre os tempos do GSO nos valores referentes à $\mathrm{PaCO}_{2}$ em relação ao basal. Embora o GSO não tenha sido suplementado com oxigênio, não há indícios de que tenha ocorrido hipoventilação, visto que os valores de $\mathrm{PaCO}_{2}$ encontram-se dentro dos limites aceitáveis (35$45 \mathrm{mmHg}$ ) para cães sob anestesia geral (Tabela 1). No GCO, foi constatado um aumento significativo, apenas no momento M1 se comparado ao basal. Esse valor pode ser explicado pelo valor de um único animal, que apresentou $\mathrm{PaCO}_{2}$ de 53,9mmHg nesse momento. Não foram observadas diferenças significativas entre os grupos analisados.

$\mathrm{Na}$ análise dos valores de $\mathrm{f}, \mathrm{PaO}_{2}, \mathrm{PaCO}_{2} \mathrm{e}$ $\mathrm{SaO}_{2}$ de ambos os grupos, pode-se afirmar que não houve depressão respiratória em decorrência dos fármacos e das doses utilizadas pela via epidural, bem como da presença ou ausência de suplementação de oxigênio. No entanto, ressalta-se que, imediatamente após a intubação, os animais do GCO foram suplementados com oxigênio, e os do GSO permaneceram apenas intubados (M1). Esse momento coincide com o pico plasmático do tiopental, fármaco reconhecido como potente depressor respiratório, contribuindo, dessa forma, para obtenção de menores valores de $\mathrm{PaO}_{2}$ no grupo GSO; pela mesma razão, foram observados redução nos valores de $\mathrm{pH}$ e aumento na $\mathrm{PaCO}_{2}$, em GSO. Além disso, soma-se a esse efeito a intensa sedação obtida com o uso da MPA proposta.

Ocorreu redução nos valores de $\mathrm{pH}$, no grupo GSO, de M2 até M6 e redução em M1, no GCO, quando comparados com M-15. Na análise entre os grupos, foram constatados valores menores de $\mathrm{pH}$ em GCO em relação ao GSO, nos momentos M0 e M1 (Tabela 1). A diminuição do pH no GCO coincidiu com a administração do tiopental, culminando com o aumento da $\mathrm{PaCO}_{2}$. Esses dados estão de acordo com os descritos por THURMON et al. (2007), os quais relatam que os barbitúricos possuem ação depressora sobre o centro respiratório bulbar, causando hipoventilação, elevação da $\mathrm{PaCO}_{2}$ e consequentemente redução do $\mathrm{pH}$. No GSO, a queda do $\mathrm{pH}$ iniciou após a epidural, perdurando até o término do período de avaliação; no entanto, os valores encontram-se dentro do intervalo fisiológico (7,36 a $7,45)$ para o sangue arterial na espécie canina (WILLIAMS et al., 1997). 


\section{CONCLUSÕES}

Conclui-se que a anestesia epidural lombossacra com morfina e lidocaína na dose e no volume propostos é efetiva para realização de OSH em cadelas, com mínimas alterações cardiovasculares e hemogasométricas, as quais são bem toleradas em animais hígidos. Essa prática é exequível em campanhas de castração em que não há possibilidade de oxigenação dos animais.

\section{FONTE DE AQUISIÇÃO}

a-Isoforine, Cristália Produtos Químicos e Farmacêuticos, Itapira, SP.

b-Lidocaína 2\% sem vasoconstritor, Cristália Produtos Químicos e Farmacêuticos, Itapira, SP.

c-DIXTAL 2010 - Dixtal Brasil Ind. e Comércio Ltda, Manaus, AM, Brasil.

d-Rapidlab 348 Bayer, São Paulo, SP, Brasil.

e-Acepromazina 1\%, Univet, São Paulo, SP.

f-Cloridrato de Petidina 50 $\mathrm{mg} \mathrm{ml}^{-1}$, Cristália Produtos Químicos e Farmacêuticos, Itapira, SP.

g-Tiopental sódico 1g, Cristália Produtos Químicos e Farmacêuticos, Itapira, SP.

h-Dimorf $5 \mathrm{mg} \mathrm{ml-1}$, Cristália Produtos Químicos e Farmacêuticos, Itapira, SP

i-Fentanil $50 \mu \mathrm{g}$ mL-1, Hipolabor, Sabará, MG

\section{COMITÊ DE ÉTICA E BIOSSEGURANÇA}

Este estudo foi aprovado pelo Comitê de Ética e Bem-estar Animal (CETEA) da Universidade do Estado de Santa Catarina (Protocolo no 1.09/07).

\section{AGRADECIMENTOS}

À Universidade do Estado de Santa Catarina, pela concessão de bolsa de Iniciação Científica.

\section{REFERÊNCIAS}

BAILEY, C.S. et al. Spinal nerve root origins of the cutaneous nerves of the canine pelvic limb. American Journal of Veterinary Research, v.49, n.1, p.115-119, 1988. Disponível em: <http://www3.interscience.wiley.com/journal/119986320/ abstract?CRETRY $=1 \&$ SRETRY $=0>$. Acesso em: 30 set. 2009. doi: $10.1111 /$ j.1439-0264.1992.tb00315.

BONATH, K.H.; SALEH, A.S. Long term pain treatment in the dog by peridural morphine. In: INTERNATIONAL CONGRESS OF VETERINARY ANESTHESIA, 1985, California. Proceedings... California: American College of Veterinary Anesthesiologists, 1985. p.7-10

BRANSON, K. R. et al. Duration of analgesia induced by epidurally administered morphine and medetomidine in dogs. Journal of Veterinary Pharmacology and Therapeutics, v.16, n.3, p.369372, 1993. Disponível em: <http://www3.interscience.wiley.com/ journal/119300330/abstract $>$. Acesso em: 30 set. 2009. doi 10.1111/j.1365-2885.1993.tb00184.
CARPENTER, R.L. et al. Incidence and risk factors for side effects of spinal anesthesia. Anesthesiology, v.76, p.906916, 1992. Disponível em: <http://journals.lww.com/ a n e sthesiology/A b s tract/1992/06000/ Incidence_and_Risk_Factors_for_Side_Effects_of.6.asp $\mathrm{x}>$. Acesso em: 30 set. 2009.

CASSU, R.N. et al. Anestesia epidural com lidocaína isolada e associada ao fentanil para realização de ováriossalpingohisterectomia em cadelas. Arquivo Brasileiro de Medicina Veterinária e Zootecnia, v.60, n.4, p. 825-831, 2008. Disponível em: <http://www.scielo.br/scielo.php?pid=S0102$09352008000400008 \&$ script $=$ sci arttext\&tlng $=\mathrm{es}>$. Acesso em: 30 set. 2009 . doi: $10.1590 / \overline{\mathrm{S}} 0102-09352008000400008$.

CRUZ, M.L. et al. Epidural anesthesia lignocaine, bupivacaine or a mixture of lignocaine and bupivacaine in dogs. Journal of Veterinary Anaesthesia, v.24, n.1, p.30-32, 1997. Disponível em: <http://www3.interscience.wiley.com/journal/ 119831742/abstract $>$. Acesso em: 30 set. 2009. doi: 10.1111/ j.1467-2995.1997.tb00265.

DINIZ, S. Animal livre. Acesso em: 17 mar. 2009. Online. Disponível em: $<$ http://animalivre.uol.com.br/home/?tipo=noticia\&id=1321>.

FARVER, T.B. et al. Cardiopulmonary effects of acepromazine of the subsequent administration of ketamine in the dog. Journal of Veterinary Anaesthesia, v.47, p.631-635, 1986.

GASPARINI, S.S. et al. Anestesia epidural com ropivacaína, lidocaína ou associação de lidocaína e xilazina em cães. Efeitos cardiorrespiratório e analgésico. Ciência Rural, v.37, p.418424, 2007. Disponível em: <http://www.scielo.br/pdf/cr/v37n2/ a19v37n2.pdf $>$. Acesso em: 30 set. 2009. doi: 10.1590/S010384782007000200019

HALL, L.W.; CLARKE, K.W. Principles of sedation, analgesia and premedication. Veterinary anaesthesia. 9.ed. Londres: Bailiière Tindall, 1991. Cap.4, p.51-79.

ISHIY, H.M. et al. Uso da lidocaína isolada ou associada à quetamina ou ao butorfanol, em anestesia epidural em cadelas submetidas à ovariosalpingohisterectomia. Revista Brasileira de Ciência Veterinária, v.9, n.1, p.134-136, 2002.

MATSUKAWA, T. et al. Heat flow and distribuition during epidural anesthesia. Anestesiology, v.83, p.961-967, 1995. Disponível em: <http://journals.lww.com/anesthesiology/pages/ articlevieweraspx ?year $=1995 \&$ issue $=11000 \&$ article $=00008 \&$ type $=$ fulltext $>$. Acesso em: 30 set. 2009.

MUIR III, W.W. et al. Manual de anestesia veterinária. 3.ed. Porto Alegre: Artmed, 2001. 432p.

NOLTE, J.G. et al. Cardiovascular effects of epidural blocks in dogs. Journal of Small Animal Practice v.24, p.17-21, 1983.

PASCOE, P.J. Advantages and guidelines for using epidural drugs for analgesia. Veterinary Clinics of North American: Small Animal Practice, v.22, n.2, p.421-423, 1992.

PASCOE, P.J.; DYSON, D.H. Analgesia after lateral thoracotomy in dogs. Epidural morphine vs. intercostals bupivacaine. Veterinary Surgery, v.22, p.141-147, 1993. Disponível em: <http:// bases.bireme.br/cgi-bin/wxislind.exe/iah/online/?IsisScript=iah/ 
iah.xis \&next Action $=1 \mathrm{nk} \&$ base=MEDLINE_19661996\& exprSearch=8511848\&indexSearch=UI\&lang $=\mathrm{i}>$. Acesso em: 30 set. 2009 .

POPILSKIS, S. et al. Prolonged postsurgical analgesia: effects epidural fentanyl infusion in dogs. In: WORLD CONGRESS OF VETERINARY ANAESTHESIA, 2000, Berne. Proceedings... Berne: ECVA, 2000. p.77.

THURMON, J.C. et al. Lumb \& Jones' veterinary anaesthesia and analgesia. 4.ed. IOWA: Blackwell, 2007. 1096p.

TORSKE, K.E. et al. End tidal halothane concetration and postoperative analgesia requirements in dogs: A comparison between intravenous oxymorphone and epidural bupivacaine alone and in combination with oxymorphone. Canadian Journal of Anaesthesia Veterinary, v.39, p.361-369, 1998. Disonível em: <http://www.pubmedcentral.nih.gov/ picrender.fcgi artid $=1539403 \&$ blobtype $=p d f>$. Acesso em: 30 set. 2009.

VALADÃO, C.A.A. et al. Administração epidural de opióides em cães: revisão bibliográfica. Ciência Rural, v.32, p.347-335, 2002. Disponível em: $<\mathrm{http}: / / w w w . s c i e l o . b r / s c i e l o . p h p ?$ pid=S0103-
$84782002000200028 \&$ script $=$ sci_arttext\&tlng $=\mathrm{e}>$. Acesso em: 30 set. 2009 . doi: $10.1590 / \mathrm{S} 0103-84782002000200028$.

VALVERDE, A. et al. Epidural morphine reduces halothane MAC in the dog. Canadian Journal of Anaesthesia Veterinary, v.36, n.6, p.629-633, 1989. Disponível em: $<$ http://www.springerlink.com/content/q7230rwm71638518/>. Acesso em: 30 set. 2009. doi: 10.1007/BF03005412.

WATERMAN, A. Accidental hypothermia during anaesthesia in dogs and cats. Veterinary Record, v.96, n.14, p.308-313, 1975.

WETMORE, L.A.; GLOWASKI, M.M. Epidural analgesia in veterinary critical care. Clinical Techiniques in Animal Small Practice, v.15, p.177-188, 2000. Disponível em: <http://www.sciencedirect.com/ science?_ob=ArticleURL\&_udi=B 75BB-4GK2NBB-

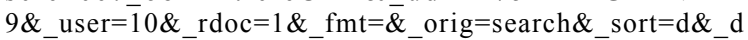
ocanchor $=\&$ view $=\mathrm{c} \&$ acct $=\overline{\mathrm{C}} 000050221 \&$ _version $=$ $1 \&$ u r l V e r s i o $\mathrm{n}=0 \& \&_{-}$u s e r i d = $10 \& \mathrm{~m}$ $\mathrm{d} 5=55 \bar{b} \mathrm{~b} 4 \mathrm{cdfd} 3 \mathrm{~d} 94513 \mathrm{~b} 710 \mathrm{a} 2286526475 \mathrm{a}>$. Acesso em: 30 set. 2009. doi: $10.1053 /$ svms.2000.16545.

WILLIAMS, E.M. et al. Oxygen transport with oscillations of inspired oxygen concentration. Respiratory physiology, v.108, p.79-87, 1997. 